\title{
Piezoelectric impact force sensor array for tribological research on rigid disk storage media
}

\author{
G.J. Burger, T.S.J. Lammerink, J.H.J. Fluitman \\ MESA Research Institute, University of Twente, P.O.Box 217, 7500 AE Enschede, the Netherlands \\ Tel: XX-31-53 892 805, Fax: XX-31-53 309547 \\ S. Imai, \\ Mechanical Engineering Research Laboratory, Hitachi, Ltd., 502, Kandatsu, Tsuchiura, Ibaraki 300, Japan \\ M. Tokuyama, S. Hirose \\ Data Storage \& Retrieval System Division, Hitachi, Ltd., 2880 Kozu, Odawara, Kanagawa 256, Japan
}

\begin{abstract}
This paper presents a method to measure impact forces on a surface by means of a piezoelectric thin film sensor array. The output signals of the sensor array provide information about the position, magnitude and wave form of the impact force. The sensor array may be used for tribological studies to the slider disk interface of a rigid disk storage device. In such a device a slider head assembly is flying above the rotating disk with a typical spacing of $100 \mathrm{~nm}$. Possible mechanical interactions between the slider and the disk are expected to produce impact forces in the order of $0.1 \mathrm{~N}$ with a frequency range from $100 \mathrm{kHz}$ to $100 \mathrm{MHz}[1]$.
\end{abstract}

\section{Introduction}

In rigid disk data storage devices a read/write head is mounted on a slider which is placed on the disk. During disk rotation an air bearing is formed between the slider and the disk (typical 100nm thick). Developments to higher storage densities lead to a lower flying height and eventually contact recording. To accomplish this, much tribological research is done to the wear characteristics of the slider/disk interface. Many different measuring methods to measure the slider behaviour, flying height and friction force are developed [2]. So far no appropriate method is found to measure the expected high frequent impulsive force peaks (impacts) at the slider disk interface. In a conventional method of measuring the impact forces a bulk piezoelectric sensor is mounted on the slider [3]. The dimensions of these sensors are rather large, therefore the eigen frequencies of the sensor lay within the interesting frequency range which makes signal interpretation difficult.

In the presented method of measuring impact forces a thin film piezoelectric sensor array is embedded in the slider. The array consists of a piezoelectric layer $(\mathrm{ZnO})$ sandwiched between a ground electrode and signal electrodes. For example in figure la, a sensor is positioned in a semi-infinite body. The impact force induces elastic waves within the body which propagate from the surface through the sensor into the material behind the sensors. If the mechanical properties of the sensor are comparable with these of the surrounding material, the sensor will hardly not affect the elastic waves and the output signal wave form will be representative for the applied impact force.

a)

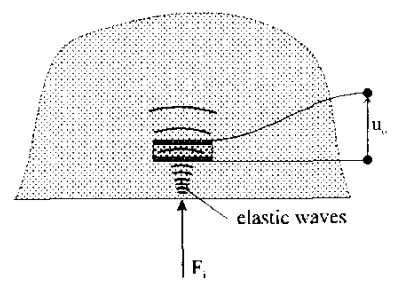

b)

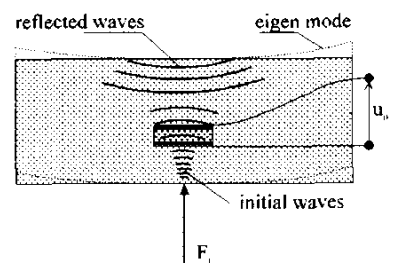

Figure 1: Principle of measuring impacts; a) impacts on a semi-infinite body; b) impacts on a finite body.

Unfortunately normal objects (like a slider) are finite and the elastic waves will reflect against the objects boundaries, as illustrated in figure $1 \mathrm{~b}$. These reflected waves shall reach the sensor in various ways and give an output signal. Eventually the waves will trigger the eigen frequencies of the object and these eigen movements will also produce an output voltage. If the sensor array is positioned close to the surface at which the impact is applied the initial waves produce the largest deformation of the sensor and therefore the maximum output voltage. The reflected waves are assumed to be lower because 
they are spread out three dimensionally. The output signal due to the eigen movements of the object may be large if the eigen modes are triggered by sequential impacts, however the wave form (or better spectrum) of these signals are predictable and can be compensated.

\section{Sensor model ${ }^{1}$}

We assume that slider/disk interactions induce elastic waves which, in vicinity of the sensor, can be regarded as one dimensional (z-axes) compressive plane waves. The system can be modelled as shown in figure 2 .

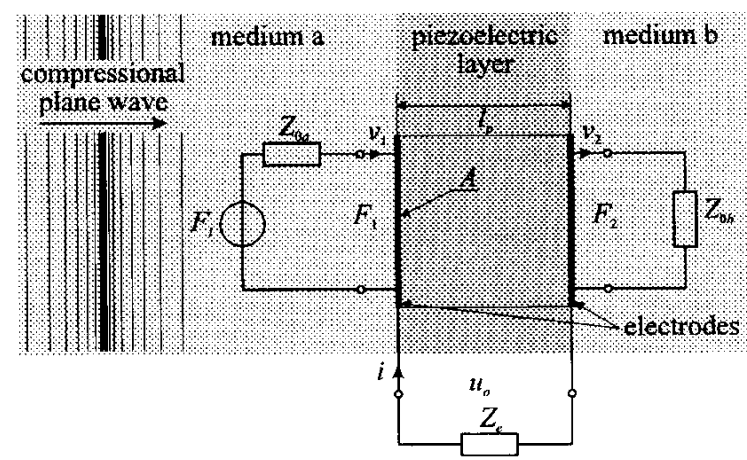

Figure 2: Physical model of a piezoelectric sensor for measuring elastic plane waves.

The compressional plane wave is propagated from the place of impact through medium a and the piezoelectric layer into medium $b$. The piezoelectric layer is modelled as a three port buffer with two mechanical ports, respectively, $\left(F_{1}, v_{1}\right)$ for the left electrode and $\left(F_{2}, v_{2}\right)$ for the right electrode, and an electrical port $\left(u_{o}, i\right)$. The stress field in the wave induces a force on the left electrode of the sensor. This in modelled by a force source $\left(F_{i}\right.$, being the integral of compressive stress, $T_{z z}$, over the sensor area $\left.\mathrm{A} ; F_{i}=T_{z} A\right)$ in series with the mechanical characteristic impedance of medium a $\left(Z_{0}\right)$. The right electrode of the sensor is loaded by the mechanical impedance of medium $\mathrm{b}\left(Z_{0_{b}}\right)$, The electrical load $\left(Z_{e}\right)$ is the input impedance of the measuring equipment. The constitutive relations in the frequencydomain for this system are [4]:

$$
\begin{aligned}
& F_{1}=F_{i}-v_{1} Z_{0 a} \\
& F_{2}=v_{2} Z_{0 b} \\
& u_{o}=-i Z_{e}
\end{aligned}
$$

\footnotetext{
${ }^{1}$ Parameters which are not defined in the text and a list of used material constants, can be found in the nomenclature at the end of this paper
}

$$
\left[\begin{array}{l}
F_{1} \\
F_{2} \\
u_{o}
\end{array}\right]=\left[\begin{array}{ccc}
\frac{Z_{0 p}}{j \tan k_{p} l_{p}} & \frac{-Z_{0 p}}{j \sin k_{p} l_{p}} & \frac{h_{33}}{j \omega} \\
\frac{Z_{0 p}}{j \sin k_{p} l_{p}} & \frac{-Z_{0 p}}{j \tan k_{p} l_{p}} & \frac{h_{33}}{j \omega} \\
\frac{h_{33}}{j \omega} & \frac{-h_{33}}{j \omega} & \frac{1}{j \omega C_{0}}
\end{array}\right] \cdot\left[\begin{array}{c}
v_{1} \\
v_{2} \\
i
\end{array}\right]
$$

Where $Z_{0_{p}}$ is the mechanical characteristic impedance, $k_{p}$ the wave number, $l_{p}$ the thickness, $C_{0}=\varepsilon_{33}^{S} A l_{p}$ the electric capacity and $h_{33}$ the piezoelectric constant of the piezoelectric layer. The set of equations (1-4) can be solved analytically, and the transfer function $H(f)=$ $u_{o} / F_{i}$ can be found. Figure 3 , gives a typical bode diagram of the transfer function.

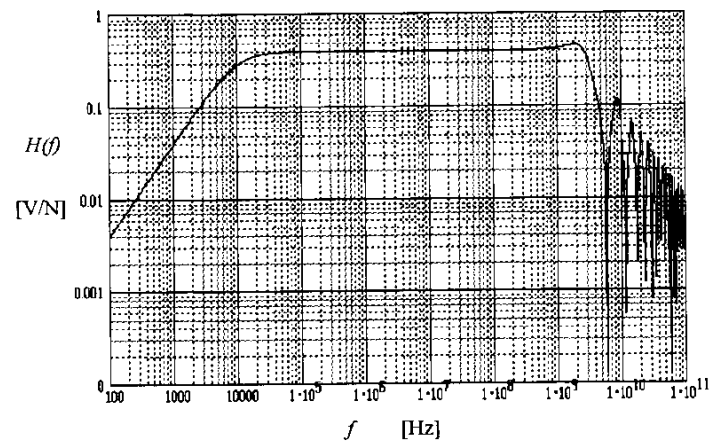

Figure 3: Transfer function $H(f)=u_{o} / F_{i}$ of a $\mathrm{ZnO}$ piezoelectric sensor element with: $l_{p}=1 \mu \mathrm{m}$ and $A=100 \times 100 \mu \mathrm{m}^{2}$, surrounded by silicon.

It can be seen that the transfer function has a large flat region. In this region the output voltage is proportional to the applied force. At high frequencies the sensors eigen frequencies may be triggered if the mechanical impedance of the piezo material is different from the mechanical impedance of the surrounding material. In case of zinc oxide and silicon no sensor resonance will occur. The dips in the bode diagram are because the voltage is an integration of the electric field. This integration yields zero for frequencies of which an integer amount of periods geometrical fit in the sensor thickness. The frequency at which the peaks and dips occur is determined by the thickness of the piezoelectric layer. For the layers used in the impact sensor this frequency is well above $100 \mathrm{MHz}$. At low frequencies the product $k_{p} l_{p}$ approaches zero, this yields the following impedance equation for the piezoelectric layer: 


$$
\left[\begin{array}{c}
F \\
u_{o}
\end{array}\right]=\left[\begin{array}{cc}
\frac{1}{j \omega C_{m}} & \frac{h_{33}}{j \omega} \\
\frac{h_{33}}{j \omega} & \frac{1}{j \omega C_{0}}
\end{array}\right] \cdot\left[\begin{array}{c}
\left(v_{1}-v_{2}\right) \\
i
\end{array}\right]
$$

where $F$ is the force on the sensor $\left(F=F_{1}=F_{2}\right)$ and $C_{m}=s_{33}^{D} l_{p} / A$ the mechanical capacity. The piezo sensor can now be considered as a ideal spring. The forces acting on both sides of the piezo material equal each other. Actually by taking the limit of $k_{p} l_{p}$ to zero we ignore the mass of the piezo material.

For practical use the sensor can be described by a voltage source $\left(\boldsymbol{u}_{p}\right)$ with an output capacity $\left(C_{p}\right)$ in series, see figure 4 . Where the electrical load is a resistor in parallel with a capacity. The capacity $\left(C_{e}\right)$ includes parasitic capacity of the signal tracks on the chip and from the chip to the measuring equipment and the input capacity of the measuring equipment. The resistor $\left(R_{e}\right)$ represents the input resistance of the measuring equipment.

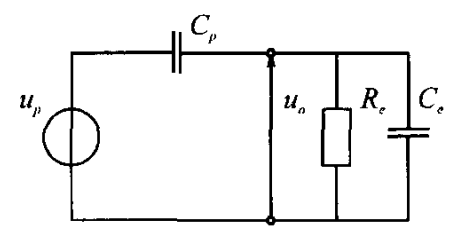

Figure 4: Electric scheme of an impact sensor element.

The voltage $\left(u_{p}\right)$ is proportional to the force applied over the sensor can be deduced from eq. (1-3) and (5).

$$
u_{p}=h_{33} C_{m} \cdot \frac{Z_{0_{b}}}{Z_{0_{a}}+Z_{0_{b}}} \cdot F_{i}
$$

The output capacity of the sensor can be calculated with eq. (7).

$$
C_{p}=\frac{C_{0}}{\left(1-h_{33}{ }^{2} C_{m} C_{0}\right)}
$$

For a typical sensor with an area of $100 \times 100 \mu \mathrm{m}^{2}$ and a thickness of $1 \mu \mathrm{m}$, surrounded by silicon, the output voltage $\left(u_{p}\right)$ will be 5 Volt per unit force $\left(u_{p} / F_{s}=5 \mathrm{~V} / \mathrm{N}\right)$ and the output capacity $\left(C_{p}\right)$ is $0.83 \mathrm{pF}$. The voltage is rather large and seems easy to measure, however the output capacity is very small therefore any parasitic capacity will decrease the sensitivity drastically.
The measured output voltage $\left(u_{o}\right)$ is given by $(8)$.

$$
u_{o}=u_{p} \cdot \frac{j \omega R_{e} C_{p}}{\left(1+j \omega R_{e}\left(C_{p}+C_{e}\right)\right)}
$$

It can be seen that the input resistance of the measuring equipment and the summation of the sensor capacity and the parasitic capacity create a differentiating behaviour at low frequencies. For typical values of the resistance and the capacities this will yield a cut-off frequency of $15 \mathrm{kHz}\left(R_{e}=1 \mathrm{M} \Omega, C_{e}=10 \mathrm{pF}, C_{p}=0.83 \mathrm{pF}\right)$.

In the desired frequency range $(100 \mathrm{kHz}-100 \mathrm{MHz})$ the output voltage will be proportional to the applied force but decreased by the ratio $C_{p} /\left(C_{p}+C_{e}\right)$. For the used typical values the output voltage will be 0.38 Volt per unit force $\left(u_{d} / F_{s}=0.38 \mathrm{~V} / \mathrm{N}\right)$. This shows the importance of keeping the parasitic capacity as low as possible.

In the above mentioned calculations it was assumed that the signals from the sensor elements are measured with a typical voltage measurement. In this case the input resistance of the measuring equipment is high and the low cut-off frequency is below the desired frequency band. An other way of measuring the signals is a current measurement. In this case the input resistance of the measuring equipment is low and the low cut-off frequency is higher than the desired frequency band. In the frequency band the desired signal $\left(F_{i}\right)$ will be differentiated. By integrating the current the original signal will be obtained. The advantage of a current measurement is that the parasitic capacity does not influence the output signal and therefore a higher signal may be obtained.

\section{Stress distribution}

For a good estimation of the signals from the sensor elements after an impact on the surface of the object a dynamical model of the stress distribution in the object seems indispensable. However, an estimation of the stress distribution can be made with the statical analysis of Timoshenko $[8, \mathrm{p} 398-420]$ for such a problem. Timoshenko deduced for the normal stress component in the z-direction $T_{z}$, the following equation:

$$
T_{z}=-\frac{3 F}{2 \pi} z^{3}\left(r^{2}+z^{2}\right)^{-5 / 2}
$$

Were $F$ is the applied force at the surface, $z$ is the distance to the surface and $r$ is the projected distance to 
the force exertion point. In the case of a distributed load the produced stresses can be found by superposition. However, if the distance to the point of application of the load is large in comparison to the radius of the load area, eq. (9) remains valid. The average force on a sensor element can be found by integration of eq. (9) over the sensor area.

\section{Experiments}

The principle of measuring impacts with thin film piezoelectric sensor arrays is evaluated. Figure 5 shows a cross sectional view of the realised test samples. The numbers between brackets refer to the process sequence. For these samples the silicon substrate is used for the ground electrode and aluminium is used for the signal electrodes. The silicon is connected via the aluminium

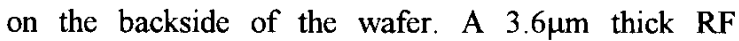
magnetron sputtered $\mathrm{ZnO}$ layer is used for the piezoelectric transduction. The $\mathrm{SiO}_{2}$ layer provides for a good substrate layer for the $\mathrm{ZnO}$ [5] and has no critical influence on the measuring principle. A typical sensor area is $500 \times 500 \mu \mathrm{m}$.

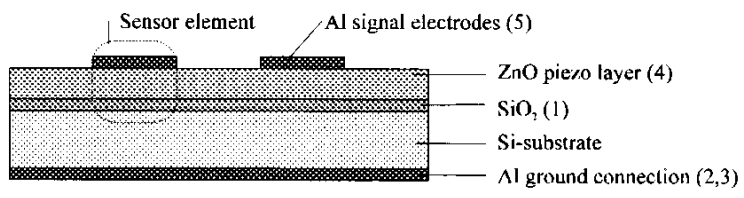

Figure 5: Schematic cross sectional view of the test samples. The numbers between brackets refer to the process sequence.

The test samples are glued on a glass rod and impact forces are exerted on the backside of the sensor chip by rolling a steel ball against the chip, see figure 6 . The elastic waves propagate from the position of impact through the sensor chip into the glass rod. This situation is comparable with a sensor in a semi-infinite body (figure la). The position of impact can be adjusted by precision translators. The impulse force can be adjusted by changing the angle or length of the trail.

Timoshenko describes a quasi-static model for an elastic collision of a ball against a flat plane [5, p420-422]. This model is used to calculate the magnitude and wave form of the induced impact force. Impact with magnitudes in the ranges of several Newton's and with a duration of several micro seconds can be realised with this set up. Signals from the sensor elements are registered with a digital oscilloscope.

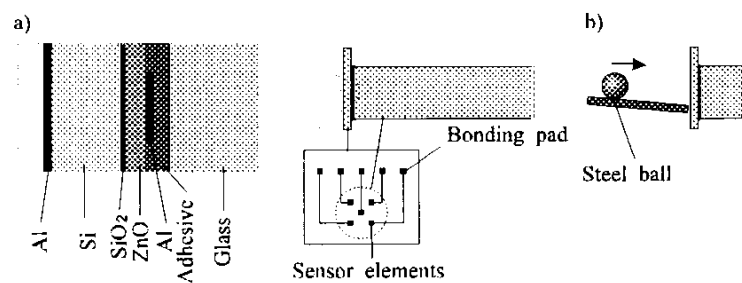

Figure 6: Experimental set up to characterise the impact sensor array.

Figure 7 shows the measured and expected output voltage $\left(u_{o}\right)$ as a function of time $(t)$. The maximum of the induced force was 8 Newton and it was applied at the centre of a the sensor element. The contact time and shape of the signal are estimated quite good by the model. The expected magnitude is lower but in the same order as the measured signal.

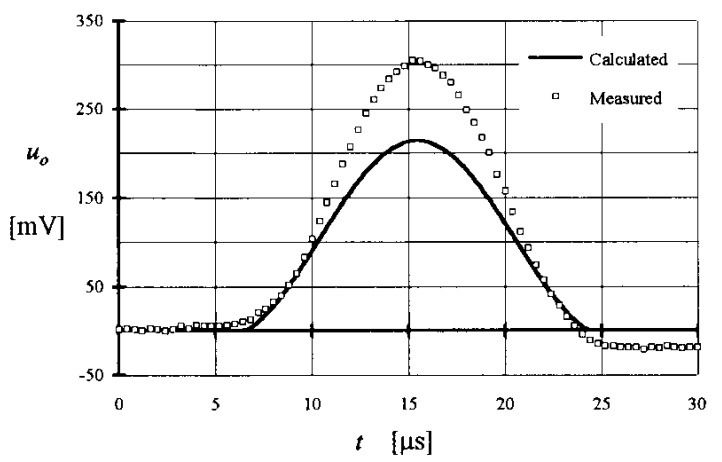

Figure 7: Measured and expected output signal $\left(u_{o}\right)$ from a sensor element after an impact. Parameters: sensor dimensions: square $500 \times 500 \mu \mathrm{m} . l_{p}=3.6 \mu \mathrm{m}, z=380 \mu \mathrm{m}$, diameter ball $=3.2 \mathrm{~mm}, h=7.5 \mathrm{~mm}, F=8 \mathrm{~N}, C_{e} \approx 60 \mathrm{pF}$

The output voltage $\left(u_{o}\right)$ as a function of the applied force on the surface $(F)$ is given in figure 8 . The position of impact is at the centre of the sensor element. For the $3.2 \mathrm{~mm}$ ball the measurements deviate from the other measurements. Possibly the position of impact was not exactly in the middle of the sensor element. The measured sensitivity $(0.1 \mathrm{~V} / \mathrm{N})$ is comparable but slightly higher that the calculations $(0.06 \mathrm{~V} / \mathrm{N})$. Further the measurements show a good linearity of the sensitivity. 


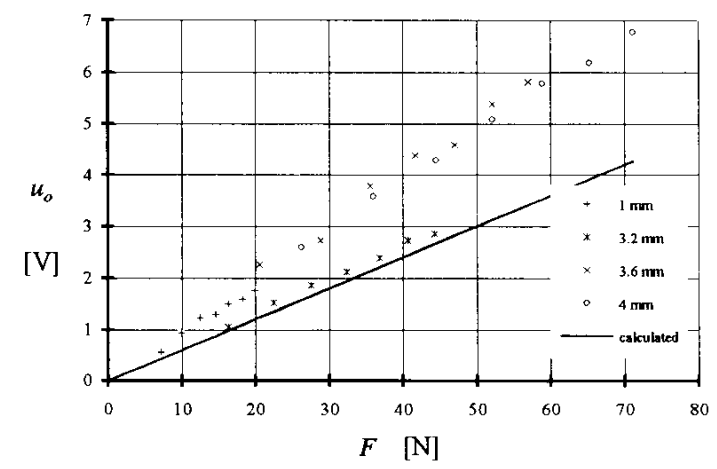

Figure 8: Measured and expected output voltage $\left(u_{o}\right)$ as a function of the applied force at the centre of a sensor element. Parameters: sensor element dimensions: square $500 \times 500 \mu \mathrm{m} . l_{p}=3.6 \mu \mathrm{m}, z=380 \mu \mathrm{m}$, diameter ball, see legend.

The output voltage $\left(\boldsymbol{u}_{o}\right)$ as function of the position of impact $\left(r_{s}\right)$ is given in figure 9 . The used sensors were $500 \mu \mathrm{m}$ wide. It can be seen that the measured sensitivity decreases to about half the maximum value if the impact is applied near the edge of the sensor elements. Figure 9 shows that if such sensor element is positioned in an array with a distance between the sensors of about $100 \mu \mathrm{m}$ both the magnitude and the exertion point of the impact can be determined with the array. The calculated sensitivity shows a wider curve than the measured curve. The neglected inertial forces in the static stress distribution model may explain this deviation.

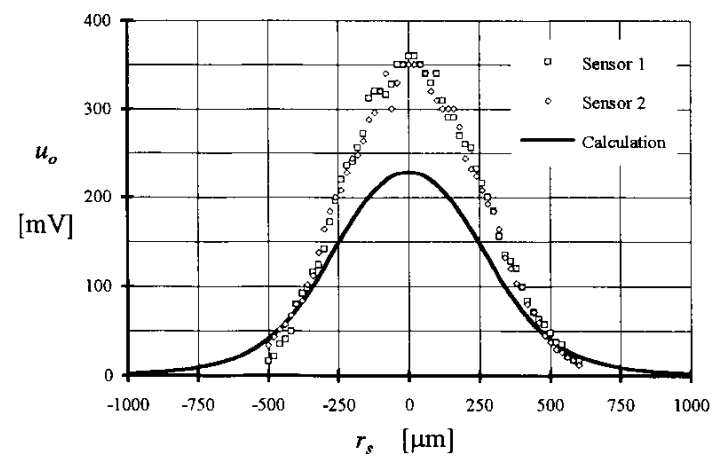

Figure 9: Measured and expected output voltage $\left(u_{0}\right)$ as a function of the position of impact relative to the centre of the sensor $\left(r_{s}\right)$. Parameters: sensor element dimensions: square $500 \times 500 \mu \mathrm{m} . \quad l_{p}=3.6 \mu \mathrm{m}, \quad z=380 \mu \mathrm{m}$, diameter ball $=3.2 \mathrm{~mm}, h=7.5 \mathrm{~mm}, F=8 \mathrm{~N}, C_{e} \approx 60 \mathrm{pF}$

The above described measurements with the test sample show that the thin film piezoelectric sensor array can be used to measure magnitude and insertion point of an impact force normal to a surface. The sensitivity of the realised sensors is about $0.1 \mathrm{~V} / \mathrm{N}$. The statical model used for an estimation of the output voltage is in fairly good agreement with the measured signals. Estimations predict the order of magnitude, but the measured signals are somewhat higher. The shape of the expected and measured signals are in good agreement which indicates a proportional relation between the applied force and the output voltage of the sensor elements.

\section{Application}

A prototype slider which is carrying the impact sensor is realised. Figure 10 shows an illustration of the realised sensor sliders. The slider consists of a rectangular body with two rails at the bottom. The bottom surface of the rails is called the "air bearing surface" (abs). This is the surface on which the slider "slides". On the front side of the slider the air bearing surface has a "taper step". This taper step is necessary for the generation of a pressure on the slider inflow side. The flying characteristics are determined by the shape of the rails and air bearing. The shape of the realised rail system is similar as the rail system of a conventional slider. The sensor array (a $2 \times 2$ matrix) is realised on the other side of the wafer. The array is positioned above the rear side of the sliding rails which has the lowest flying height. A glass strip is mounted on the wafer covering the rear part of the slider and leaving the electrical bonding pads open.
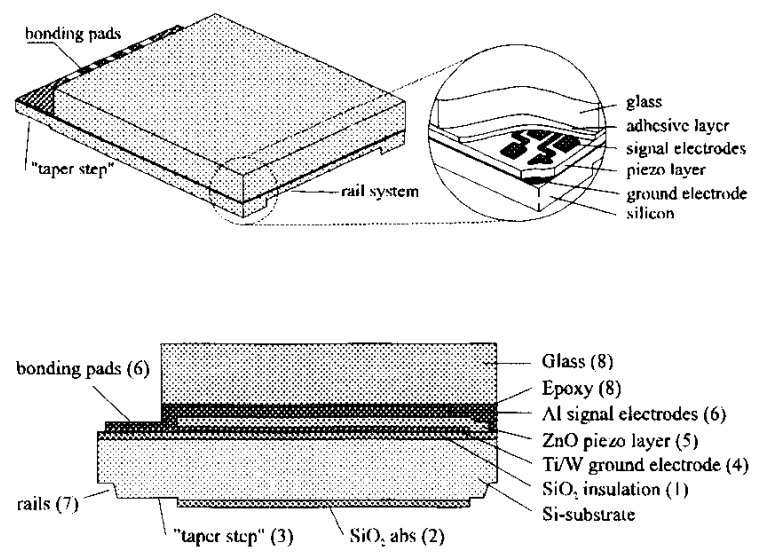

Figure 10: a) Impression of the realised prototype sensor slider. b) Cross sectional view. The numbers between brackets refer to the process sequence.

In figure 11 an illustration of the realised slider that is bonded to a suspension is shown. The electrical connections are ultrasonic bonded from the slider to the preamplifier. 


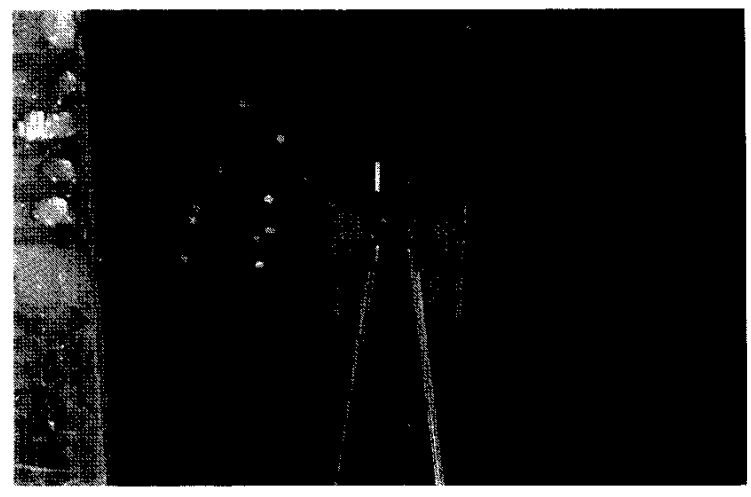

Figure 11: A prototype sensor slider mounted to the suspension.

The flying height of the slider is measured with an interferometer. At a rotation frequency of $3600 \mathrm{rpm}$ and a radius of $58 \mathrm{~mm}$ the flying height was $480 \mathrm{~nm}$ at the front side of the slider and $120 \mathrm{~nm}$ at the rear side. This is comparable to the flying characteristics of a conventional slider (respectively $500 \mathrm{~nm}$ and $130 \mathrm{~nm}$ ). To test the sensors an obstacle is realised on the disk by a scratch with a knife. This yields two ridges of about $300 \mathrm{~nm}$ high and $140 \mathrm{~nm}$ apart. Figure 12 . shows a typical amplified $(60 \mathrm{~dB}, 1000 \times)$ signal from a thin film sensor element in the sensor slider after passing the obstacle. The input capacity of the amplifier was high therefore a large amplification was needed. The signal is in the order of tens of millivolts (after amplification $0.4 \mathrm{~V}$ ) and the signal noise ratio is larger than in the conventional method. The laser Doppler vibrometer (LDV) output, which is used as a reference signal for the position of the obstacle on the disk, indicates that the piezo output signal is indeed a result of the slider disk interaction. The time difference of the two signals is caused by a slightly different measuring position of the LDV and the sensor slider.

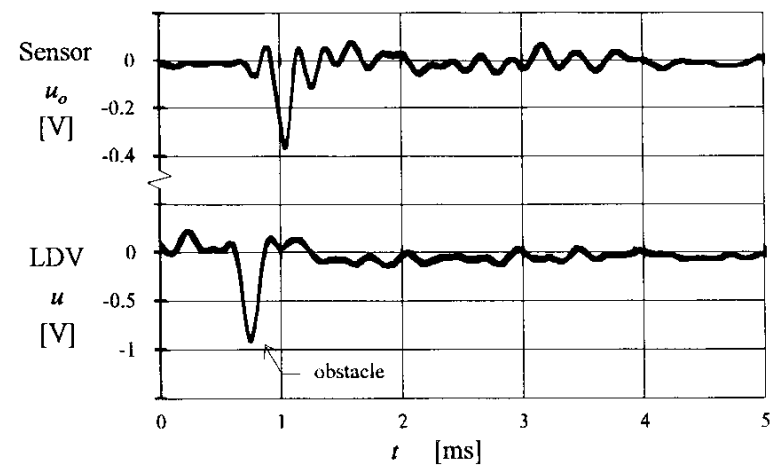

Figure 12: Amplified signal from a thin film piezo element the sensor slider after passing an obstacle on the disk. amplification: $60 \mathrm{~dB}(1000 \times)$

\section{Conclusions}

A piezoelectric impact sensor array is presented with which the amplitude, wave form and exertion point of an impact force can be measured. The feasibility of measuring impacts at the air bearing surface of a magnetic recording slider has been shown.

\section{References}

[1] H.L. Leo and G.B. Sinclair, "So how hard does a slider hit a disk?", IEEE Transactions on Magnetics, 27:6 (1991) p5154-5156.

[2] B. Bhushan, Tribology and mechanics of magnetic storage devices, Springer-Verlag, New York, (1992).

[3] K. Mochizuki, I. Sato and T. Hayashi, "Detection of an impulse force in head-disk media contact using small piezoelectric transducers", Journal of Acoustic emission, 8:3 (1989) p35-39.

[4] B.A. Auld, Acoustic fields and waves in solids, Vol 1. John Wiley \& Sons, New York, (1973).

[5] S.P. Timoshenko and J.N. Goodier, Theory of Elasticity, McGraw-Hill Book Company, (1934).

[6] F.S. Hickernell, Zinc oxide films for acoustoelectric device applications, IEEE Transactions on Sonics and Ultrasonics, SU-32 (1985) 621-629.

\section{nomenclature:}

material constants:

$\varepsilon_{33}^{S}$ : dielectric constant at constant strain

$s_{33}^{D}$ : compliance at constant dielectric displacement

$h_{33}$ : piezoelectric constants:

subscripts:

33: compressive in z-direction

$Z_{o}$ : characteristic mechanical impedance:

$$
Z_{0_{a}}=A \sqrt{\frac{\rho_{a}}{s_{33_{a}}}} ; \quad Z_{0_{b}}=A \sqrt{\frac{\rho_{b}}{s_{33_{b}}}} \quad Z_{0_{p}}=A \sqrt{\frac{\rho_{p}}{s_{33_{p}}^{D}}}
$$

$k_{p}$ : wave number:

$$
k_{p}=\omega \sqrt{\rho_{p} s_{33_{p}}^{D}}
$$

\begin{tabular}{|l|c|c|c|c|}
\hline \multicolumn{5}{|c|}{ Material properties $[4]$} \\
\hline & $\varepsilon_{33}^{s}$ & $h_{33}$ & $s_{33}^{D}$ & $\rho$ \\
\hline & $\cdot 10^{-11}[\mathrm{~F} / \mathrm{m}]$ & $\cdot 10^{10}[\mathrm{~N} / \mathrm{C}]$ & $\cdot 10^{-12}\left[\mathrm{~m}^{2} / \mathrm{N}\right]$ & {$\left[\mathrm{kg} / \mathrm{m}^{3}\right]$} \\
\hline $\mathrm{ZnO}$ & $7.242^{1}$ & 1.82 & 5.49 & 5660 \\
\hline Silicon & 10.3 & - & 7.68 & 2332 \\
\hline
\end{tabular}

